\title{
Effects of a Verbal Intervention for Head and Neck Posture on Lumbar Lordosis Angles in Healthy Young and Older Adults: a Preliminary Study
}

Meiling Zhai

Tianjin University of Sport https://orcid.org/0000-0001-7444-0218

Yongchao Huang

Nanjing Sport Institute

Shi Zhou

Southern Cross University

Jiayun Feng

Tianjin University of Sport

Chaolei Pei

Shanxi Normal University

Li Wen ( $\nabla$ wenli34@hotmail.com )

Nanjing Sport Institute

\section{Research Article}

Keywords: postural study, lumbar lordosis angle, spine, position, age

Posted Date: January 3rd, 2022

DOI: https://doi.org/10.21203/rs.3.rs-1194944/v1

License: () (1) This work is licensed under a Creative Commons Attribution 4.0 International License.

Read Full License 


\section{Abstract}

Background Postural rehabilitation plays an important role in the treatment of non-specific low back pain. Although pelvic inclination has been widely used to improve lumbar lordosis, the effect of cervical anterior inclination on lumbar lordosis in young and older adults, in sitting and standing posture is still unclear. This preliminary study was designed to examine the influence of changing the cervical anterior angle on the lumbar lordosis angle, through alterations of the head position under the natural sitting and standing conditions, aiming to provide a basis for establishing a new postural rehabilitation strategy.

Methods Thirty-eight older ( $68.4 \pm 5.9$ years old $)$ and 36 young $(24.0 \pm 2.2$ years old $)$ healthy adults participated in this study. The four spinal regional angles - cervical anterior angle, thoracic kyphosis angle, lumbar lordosis angle, and pelvic foreword inclination angle were measured in standing and relaxed sitting postures to determine the effects of a postural cueing, "inclining head backward and performing chin tuck", for the head and neck posture on lumbar lordosis angle.

Results In the standing posture, the pelvic foreword inclination angle in the older group was significantly smaller $(P<0.001)$ than that in the young group, and increased significantly $(P<0.001)$ post the postural cueing. In addition, the thoracic kyphosis angle in the standing $(P=0.001)$ and sitting $(P=0.003)$ positions was significantly reduced post the postural cueing. However, the lumbar lordosis angle post postural cueing increased significantly in both the standing position $(P<0.001)$ and sitting position $(P$ $<0.001)$.

Conclusion The results suggest that increasing the cervical anterior angle can indeed increase the lumbar lordosis angle, and the cervical anterior inclination can be used as an alternative to pelvic foreward inclination to improve the lumbar lordosis angle. Furthermore, the change of head and neck posture can reduce the thoracic kyphosis angle, making it possible to establish a new non-invasive body posture rehabilitation strategy.

(approval number TJUS2019032)

\section{Background}

Lumbar lordosis (LL) has a great clinical significance because it is the basis for the treatment and prevention of low back pain (LBP) [1]. According to epidemiological studies, $50-80 \%$ of people experience an LBP at least once in their lifetime [2]. In addition, the prevalence of LBP increases with age [3].

The reduction of lumbar lordosis angle (LLA) is considered to be one of the causes of LBP [4]. Pries et al. conducted a study on symptomatic patients aged 20-75 years and showed that the older adults commonly had the phenomenon of stooping due to physical degeneration or iatrogenic factors, and the LLA of the older adults was significantly smaller than that of the young adults during standing [5]. In addition, sitting posture is also a potential cause of LBP [6]. When sitting, the pelvis rotates backward and 
the LL becomes flat. This posture increases the pressure on the passive components in the back of the spine, which in turn may cause LBP [7]. A study on 459 university students with LBP showed that the prevalence rate of LBP among the participants was $75.8 \%$, and the prevalence rate of chronic LBP was $12.4 \%$ [8]. In addition, for the elderly, due to the decline in physical function, half of their waking time is in a sitting position [9], so the sitting position in daily life is a vital contributor to LL status [10].

Therefore, when there is an abnormality in LL, how to correct it has become a practical question in research. Previous studies have shown that the pelvic forward inclination can extend the lumbar spine and increase the LLA [11]. However, Claeys et al. showed that posture correction could not be initiated by optimizing the position of the pelvis when standing [12]. In addition, individuals suffering from LBP also have an increased risk of persistent neck pain [13], and the pain is associated with weakened strength and reduced activation in the muscles acting on the shoulder and neck [14]. However, to our knowledge, little research has been reported on improving the LLA and preventing and treating lumbar injury through the correlation between the neck and lumbar.

According to the above background, the purpose of this research was to explore the effect of "inclining head backward and performing chin tuck" postural cueing on LL with the objective of reducing cervical anterior inclination. It was hypothesized that the LLA could be improved by interfering with the frontal inclination of the cervical vertebrae to adjust the body posture. The results of the research would set a basis for improving the stooping hunchback of the old people and young people's desk sitting posture, preventing and treating lower back pain, and establishing a new body posture rehabilitation strategy.

\section{Methods}

This study used a self-control design for a within-group comparison of the spine angle changes pre and post the intervention in the young group and the older group, as well as a cross-sectional design for a between-group comparison for the effect of age, to verify the effectiveness of postural cueing in improving LLA.

\section{Participants}

A total of 74 healthy participants ( 36 young adults, including 14 females and 22 males; 38 older adults, including 36 females and 2 males) volunteered to participate in this study. The young adults had an average age of $24.0 \pm 2.2$ years, weight of $67.2 \pm 11.8 \mathrm{~kg}$, height of $1.72 \pm 7.9 \mathrm{~m}$, and body mass index of $22.4 \pm 2.9 \mathrm{~kg} / \mathrm{m}^{2}$; while the older adults had an average age of $68.4 \pm 5.9$ years, weight of $61.6 \pm 8.4 \mathrm{~kg}$, height of $1.6 \pm 4.9 \mathrm{~m}$, and body mass index of $24.1 \pm 3.0 \mathrm{~kg} / \mathrm{m}^{2}$.

The participants were recruited from the local community, with the young adults were university students and the older adults were local residents of Tianjin City. All participants had no recent spinal (cervical, thoracic, or lumbar) pain or severe musculoskeletal problems in the lower extremities. The experimental procedures were approved by the Ethics Committee of Tianjin University of Sport (approval number 
TJUS2019032), and all participants gave their written informed consent prior to the commencement of the study.

\section{Spine angle assessments}

A PA200 posture evaluation system (BigSports, Japan), was used to measure the cervical anterior angle (CAA) and pelvic foreword inclination angle (PFIA). A digital camera (Sony A200 DSLR-A200K Scope DT18-70 mm F3.5-5.6; Tokyo, Japan) was placed on a tripod, with a height of $1.25 \mathrm{~m}$ and $2.41 \mathrm{~m}$ away from the participant, to take photographs of the posture in natural standing and the posture after the postural cueing.

Participants wore tight clothing. The markers for photo-image analysis were affixed over the bony landmarks on the cloth and skin, including ear foramen, spinous process of the cervical vertebra C7, greater tubercle of the humerus, anterior superior iliac spine (ASIS), and posterior superior iliac spine (PSIS).

For the natural standing position, the participants were asked to take their normal standing position, barefoot on the plantar pressure plate, with their feet $10 \mathrm{~cm}$ apart, and arms relaxed along the side of the body, with their gaze horizontally on the camera in front.

For the natural sitting position, the participants (young adult group only) were asked to sit naturally in the chair as they used in school. Using external markers in combination with digital photographs has proved to be reliable and valid in postural evaluation [15-17]. In addition, the method of taking photographs for spine angle analysis, as a part of the non-invasive whole body posture assessment, can help avoid radiographic exposure [18], replacing methods that are expensive and having health risks [19].

The participants sat in the school chair, with their arms naturally placed on the table, and their gaze horizontally on the camera in front.

The SpineScan spine tester (Sunlight, Israel) was used to measure the participants' spine angle pre and post the intervention. When measuring, the electronic spine measuring instrument was slid along the spine from above C1 to S1, and the thoracic kyphosis angle (TKA) and LLA in the natural standing and sitting position, and the CAA in the natural sitting position were measured. Studies have proved that the SpineScan is reliable to test the spine angle under the operation of the same user [20].

\section{The postural cueing}

Standing posture was assessed by photographing, with multiple pictures were taken and the best one was used in the assessment, and sitting posture was assessed by physical measurement, pre and post the postural cueing. The participants adjusted their posture according to the verbal instruction of " inclining head backward and performing chin tuck", that aimed to increase the CAA.

\section{Muscle tension simulation}


We evaluated the muscle tension simulated by computer. The program for assessing postural changes caused by muscle imbalance was set up in the PA200 posture evaluation system. According to the body posture measured in our experiment, the backward calculation was made. The simulated muscle tension changes were presented in the images of the participants (Figure 2).

Figure 1 shows the definition of all posture angles. Figure 2 is an illustration of the muscle tension estimation, which shows changes in muscle tension, and the color represents the muscle tension state, with the red color represents excessive lengthening of the muscle, blue color indicates excessive shortening of the muscle, and the muscle area without color indicates the normal muscle state.

\section{Statistical analysis}

The data was analyzed by IBM SPSS Statistics (ver.23.0, New York, USA) statistical software. Each parameter was described as the mean \pm SD. Data distribution normality was checked by applying the Shapiro-Wilk test and the equal variance was judged by the Levene's variance homogeneity test. To test the validity of the postural cueing, CAA pre and post the cueing intervention was analyzed by paired ttests. The mixed-model analysis of variance (ANOVA) was used for between-groups (age) and withingroup (intervention) comparisons. The Wilcoxon signed-rank test was used to compare the spinal angle pre and post intervention under sitting conditions in the young adult group. At the same time, Pearson correlation analysis was carried out on demographic factors such as gender and age in relation to the measured variables. To test the effect of the intervention on muscle tension, ordered Logistic regression analysis was used. Statistical significance was set at $P<0.05$. Thwith repeated measures, within-between interaction, under the assumptions of a level 0.05 , power 0.8 , and effect size 0.25 , the minimum sample size required was estimated as 34.e statistical estimation of sample size was calculated using $\mathrm{G}$ * power 3.1.9.2. Fortwo-way ANOVA

\section{Results}

\section{Analysis of the difference of spine angle under natural standing and sitting posture between the young and older adult groups}

It can be seen from Table 1 that the CAAs of the three assessments (young and older standing, and young sitting) post the postural cueing were all greater than that pre the intervention, and the difference was statistically significant $(t=5.924, P<0.001 ; t=6.291, P<0.001 ; t=5.924, P<0.001)$, indicating that the postural cueing had a significant effect on the posture. 
Table 1

Comparisons of CAA pre and post the postural cueing

\begin{tabular}{|llllll|}
\hline Group & \multicolumn{2}{l}{ CAAs (degree) } & & & \\
\cline { 2 - 6 } & $\begin{array}{l}\text { Pre the } \\
\text { intervention }\end{array}$ & $\begin{array}{l}\text { Post the } \\
\text { intervention }\end{array}$ & $\begin{array}{l}\text { Intervention (post-pre) } \\
\text { difference }\end{array}$ & $\boldsymbol{t}$ & $\begin{array}{l}\boldsymbol{P} \\
\text { value }\end{array}$ \\
\hline $\begin{array}{l}\text { Standing young } \\
(n=36)\end{array}$ & $90.67 \pm 9.67$ & $97.22 \pm 9.34$ & $6.56 \pm 6.64$ & 5.924 & $<0.001$ \\
\hline $\begin{array}{l}\text { Standing older }(n \\
=38)\end{array}$ & $91.34 \pm 9.92$ & $99.05 \pm 12.81$ & $7.71 \pm 7.55$ & 6.291 & $<0.001$ \\
$\begin{array}{l}\text { Seated young }(n \\
=36)\end{array}$ & $8.42 \pm 3.26$ & $15.14 \pm 4.64$ & $6.72 \pm 3.94$ & 10.238 & $<0.001$ \\
\hline
\end{tabular}

Table 2 shows that the interaction of age and intervention had no statistically significant effect on the spine angle $(F=0.241, P=0.625 ; F=0.029, P=0.865 ; F=1.091, P=0.300)$. The comparison between groups showed that the TKA of the elderly was larger than that of the young $(F=3.265, P=0.075)$, the LLA was smaller than that of the young $(F=2.793, P=0.099)$, but these differences were not statistically significant; and the anterior inclination angle of the pelvis was significantly larger in the older adults than that of the young people $(F=187.098, P<0.001)$. The comparison for pre and post the intervention showed that the TKA was significantly reduced $(F=12.522, P=0.001)$; the LLA was significantly increased $(F=66.571, P<0.001)$; and the PFIA significantly increased $(F=72.466, P<0.001)$. 
Table 2

Comparisons of thoracic, lumbar, and pelvic angles between the age groups and pre and post the intervention

\begin{tabular}{|c|c|c|c|c|c|c|}
\hline \multirow[t]{2}{*}{ Intervention } & \multicolumn{2}{|l|}{ TKA (degree) } & \multicolumn{2}{|l|}{ LLA (degree) } & \multicolumn{2}{|c|}{ PFIA (degree) } \\
\hline & $\begin{array}{l}\text { Young } \\
\text { group } \\
(n=36)\end{array}$ & $\begin{array}{l}\text { Oder group } \\
(n=38)\end{array}$ & $\begin{array}{l}\text { Young } \\
\text { group } \\
(n=36)\end{array}$ & $\begin{array}{l}\text { Oder } \\
\text { group } \\
(n= \\
38)\end{array}$ & $\begin{array}{l}\text { Young } \\
\text { group } \\
(n= \\
36)\end{array}$ & $\begin{array}{l}\text { Oder } \\
\text { group } \\
(n=38)\end{array}$ \\
\hline Pre-intervention & $23.28 \pm 4.11$ & $25.68 \pm 6.02$ & $9.85 \pm 5.73$ & $\begin{array}{l}7.96 \pm \\
3.14\end{array}$ & $\begin{array}{l}22.97 \pm \\
5.01\end{array}$ & $9.8 \pm 3.22$ \\
\hline Post-intervention & $21.33 \pm 6.31$ & $23.09 \pm 5.91$ & $13.31 \pm 6.52$ & $\begin{array}{l}11.28 \pm \\
5.48\end{array}$ & $\begin{array}{l}25.40 \pm \\
4.54\end{array}$ & $\begin{array}{l}12.91 \pm \\
4.17\end{array}$ \\
\hline $\begin{array}{l}\text { Comparison between } \\
\text { groups } \\
(F, P)\end{array}$ & \multicolumn{2}{|l|}{$3.265,0.075$} & \multicolumn{2}{|l|}{$2.793,0.099$} & \multicolumn{2}{|c|}{$187.098,0.000$} \\
\hline $\begin{array}{l}\text { Comparison pre and } \\
\text { post intervention } \\
(F, P)\end{array}$ & \multicolumn{2}{|l|}{$12.522,0.001$} & \multicolumn{2}{|l|}{$66.571,0.000$} & \multicolumn{2}{|c|}{$72.466,0.000$} \\
\hline $\begin{array}{l}\text { Interaction of age and } \\
\text { intervention } \\
(F, P)\end{array}$ & \multicolumn{2}{|l|}{$0.241,0.625$} & \multicolumn{2}{|l|}{$0.029,0.865$} & \multicolumn{2}{|c|}{$1.091,0.300$} \\
\hline
\end{tabular}

Table 3 shows the kyphosis angle of thoracic vertebrae in sitting position decreased significantly post postural cueing $(Z=-2.957, P=0.003)$. The postural cueing resulted in an increase of LLA in sitting position $(Z=-5.026, P<0.001)$. 
Table 3

Comparisons of TK and LL pre and post sitting posture intervention

\begin{tabular}{|c|c|c|c|c|c|}
\hline Spine angle & $\begin{array}{l}\text { Pre- } \\
\text { intervention }\end{array}$ & $\begin{array}{l}\text { Post- } \\
\text { intervention }\end{array}$ & $\begin{array}{l}\text { intervention (post- } \\
\text { pre) }\end{array}$ & $Z$ & $\begin{array}{l}\text { Significant } \\
\text { (double } \\
\text { tail) }\end{array}$ \\
\hline $\begin{array}{l}\text { TKA in sitting } \\
\text { position }\end{array}$ & 23.460 & 22.890 & -0.795 & $\begin{array}{l}-2.957 \\
b\end{array}$ & 0.003 \\
\hline $\begin{array}{l}\text { LLA in sitting } \\
\text { position }\end{array}$ & 5.540 & 8.130 & 2.380 & $\begin{array}{l}-5.013 \\
c\end{array}$ & 0.000 \\
\hline \multicolumn{6}{|c|}{ a Wilcoxon signed-rank test } \\
\hline \multicolumn{6}{|c|}{ b Based on the positive rank } \\
\hline c Based on the & e rank & & & & \\
\hline
\end{tabular}

The correlation coefficients between the spine angle and anthropometric variables such as age, height, weight, and BMI in standing and sitting positions are shown in Table 4.

Table 4

Correlation coefficients of spine angle and anthropometrical measurements of the participants in standing and sitting postures

\begin{tabular}{|llllll|}
\hline Posture & Spine angle & Age & Height & Weight & BMI \\
\hline Standing position & CAA & 0.012 & $-.351^{\star \star}$ & $-.481^{\star \star}$ & $-.336^{\star *}$ \\
\cline { 2 - 6 } & TKA & $.246^{*}$ & -0.179 & 0.019 & 0.219 \\
\cline { 2 - 6 } & LLA & -0.195 & -0.054 & -0.082 & -0.055 \\
\cline { 2 - 6 } Sitting position & PFIA & $-.848^{\star \star}$ & $.471 * \star$ & 0.126 & $-.257 *$ \\
& CAA & 0.109 & -0.190 & -0.040 & 0.133 \\
\cline { 2 - 6 } & TKA & 0.190 & -0.146 & -0.235 & -0.243 \\
\cline { 2 - 6 } & LLA & 0.150 & -0.077 & -0.108 & -0.097 \\
\hline * Representative $P<0.05 ; * *$ Representative $P<0.01$. & & \\
\hline
\end{tabular}

\section{Comparison between the older and the young groups}

Figure 3 shows that there is no significant difference in CAA between the older and the young adults' pre or post the intervention when standing naturally. The adjustment of the CAA to the spine angle is mainly reflected in the lumbar spine. Although the lordosis angle of the old lumbar spine was smaller than that of the young, the LLA of the older adult group increased significantly post the intervention. In addition, the 
TKA was also affected by the intervention on the cervical spine. Although the TKA of the older was larger than that of the young, it was significantly reduced post the intervention. However, although the anterior pelvic angle of the elderly increased significantly post the intervention, the gap was still large compared with that of the young.

Sequential logistic regression analysis was used to analyze the effect of the postural cueing of "inclining head backward and performing chin tuck" on muscle tension (muscle passive shortening, muscle normal, muscle passive elongation). The highest grade was "muscle passive shortening". The results are shown in Table 5. Post the intervention, the OR values of "passive shortening" of the left and right rhomboid muscles were 0.161 times pre the intervention $\left(X^{2}=5.072, P=0.024\right)$ and 5.939 times $\left(X^{2}=4.785, P=\right.$ $0.029)$ respectively. The OR values of "passive shortening" of left and right halibut muscles were 6.538 times pre the intervention $\left(X^{2}=5.279, P=0.022\right)$ and 6.538 times $\left(X^{2}=5.279, P=0.022\right)$. The OR value of "passive shortening" of the left quadriceps post intervention was 8.83 times greater that that in pre the intervention $\left(X^{2}=7.44, P=0.006\right)$ and for the right quadriceps it was 3.626 times greater that pre intervention $\left(X^{2}=4.128, P=0.042\right)$. However, in the older group only the left diamond muscle showed a significant change post the intervention, with the OR value of "passive shortening" post-intervention was 0.272 times greater than that of pre intervention $\left(X^{2}=5.64, P=0.018\right)$. 
Table 5

Effects of postural cueing on muscle tension in young and older people

\begin{tabular}{|c|c|c|c|c|c|c|c|}
\hline & \multirow[t]{2}{*}{ Muscle } & \multirow[t]{2}{*}{$B$} & \multirow{2}{*}{$\begin{array}{l}\text { Wader } \\
\text { Chi- } \\
\text { square }\end{array}$} & \multirow[t]{2}{*}{ Significance } & \multirow[t]{2}{*}{$\operatorname{Exp}(B)$} & \multicolumn{2}{|c|}{$\begin{array}{l}95 \% \mathrm{Cl} \text { of } \\
\operatorname{Exp}(B)\end{array}$} \\
\hline & & & & & & $\begin{array}{l}\text { Lower } \\
\text { limit }\end{array}$ & $\begin{array}{l}\text { upper } \\
\text { limit }\end{array}$ \\
\hline \multirow[t]{13}{*}{ Young } & Right trapezius & -1.079 & 2.806 & 0.094 & 0.340 & 0.096 & 1.201 \\
\hline & Left rhomboid muscle & -1.826 & 5.072 & 0.024 & 0.161 & 0.033 & 0.789 \\
\hline & Right rhomboid muscle & 1.782 & 4.785 & 0.029 & 5.939 & 1.204 & 29.311 \\
\hline & Left latissimus dorsi & 0.261 & 0.317 & 0.573 & 1.298 & 0.524 & 3.212 \\
\hline & Right latissimus dorsi & -0.261 & 0.317 & 0.573 & 0.771 & 0.311 & 1.907 \\
\hline & $\begin{array}{l}\text { Left quadratus } \\
\text { lumborum }\end{array}$ & 0.526 & 1.191 & 0.275 & 1.693 & 0.658 & 4.355 \\
\hline & $\begin{array}{l}\text { Right quadratus lumbar } \\
\text { muscle }\end{array}$ & -0.985 & 3.779 & 0.052 & 0.374 & 0.138 & 1.008 \\
\hline & Left soleus muscle & 1.878 & 5.279 & 0.022 & 6.538 & 1.318 & 32.442 \\
\hline & Right soleus muscle & 1.878 & 5.279 & 0.022 & 6.538 & 1.318 & 32.442 \\
\hline & Left iliopsoas & 1.124 & 3.010 & 0.083 & 3.077 & 0.864 & 10.954 \\
\hline & Right iliopsoas muscle & -1.124 & 3.010 & 0.083 & 0.325 & 0.091 & 1.157 \\
\hline & Left quadriceps & 2.178 & 7.440 & 0.006 & 8.83 & 1.846 & 42.233 \\
\hline & Right quadriceps & 1.288 & 4.128 & 0.042 & 3.626 & 1.047 & 12.562 \\
\hline \multirow[t]{10}{*}{ Older } & Right trapezius & -0.575 & 1.337 & 0.248 & 0.563 & 0.212 & 1.491 \\
\hline & Left rhomboid muscle & -1.303 & 5.640 & 0.018 & 0.272 & 0.093 & 0.796 \\
\hline & Right rhomboid muscle & 0.864 & 2.883 & 0.090 & 2.372 & 0.875 & 6.431 \\
\hline & Left latissimus dorsi & -0.217 & 0.205 & 0.651 & 0.805 & 0.315 & 2.059 \\
\hline & Right latissimus dorsi & 0.217 & 0.205 & 0.651 & 1.242 & 0.486 & 3.177 \\
\hline & $\begin{array}{l}\text { Left quadratus } \\
\text { lumborum }\end{array}$ & -0.318 & 0.501 & 0.479 & 0.727 & 0.301 & 1.756 \\
\hline & $\begin{array}{l}\text { Right quadratus lumbar } \\
\text { muscle }\end{array}$ & -0.468 & 1.096 & 0.295 & 0.627 & 0.261 & 1.503 \\
\hline & Right hamstring & -0.292 & 0.290 & 0.590 & 0.747 & 0.258 & 2.161 \\
\hline & Left iliopsoas & 0.256 & 0.255 & 0.613 & 1.292 & 0.478 & 3.494 \\
\hline & Left quadriceps & -0.660 & 2.359 & 0.125 & 0.517 & 0.223 & 1.200 \\
\hline
\end{tabular}




\begin{tabular}{|c|c|c|c|c|c|c|}
\hline \multirow[t]{2}{*}{ Muscle } & \multirow[t]{2}{*}{$B$} & \multirow{2}{*}{$\begin{array}{l}\text { Wader } \\
\text { Chi- } \\
\text { square }\end{array}$} & \multirow[t]{2}{*}{ Significance } & \multirow[t]{2}{*}{$\operatorname{Exp}(B)$} & \multicolumn{2}{|c|}{$\begin{array}{l}95 \% \mathrm{Cl} \text { of } \\
\operatorname{Exp}(B)\end{array}$} \\
\hline & & & & & $\begin{array}{l}\text { Lower } \\
\text { limit }\end{array}$ & $\begin{array}{l}\text { upper } \\
\text { limit }\end{array}$ \\
\hline Right quadriceps & 0.460 & 1.043 & 0.307 & 1.584 & 0.655 & 3.832 \\
\hline
\end{tabular}

\section{Comparison between sitting position and standing position}

As seen from figure 4, the kyphosis angle of thoracic vertebrae in both standing and sitting positions decreased post the intervention, but there was no significant difference between the two positions. In addition, the LLA in the sitting position was significantly smaller than that in the standing position, and the LLA increased significantly post the intervention.

\section{Discussion}

The main findings of this paper are that there was no statistical difference between the thoracic kyphosis and the lumbar lordosis in the standing position, while the pelvic forward inclination of the older group is significantly smaller than that of the young group. Thoracic kyphosis, lumbar lordosis, and pelvic forward inclination were significantly different pre and post verbal intervention. In addition, pre and post the intervention in sitting position, the thoracic kyphosis angle and lumbar lordosis angle of the young group were statistically different, and the thoracic kyphosis angle of sitting posture was near zero.

The study of muscle tension in young and older groups found that pre and post the verbal intervention, the left trapezius muscle, left hamstring muscle, left and right soleus muscle, tibialis anterior muscle, and fibula brevius muscle of the two groups showed no significant changes in muscle tension in response to the intervention. In addition, the left and right rhomboid muscles were significantly elongated in young people after the intervention compared with before intervention; the left and right soleus muscles and the left and right quadriceps were significantly shortened. However, only the left rhomboid muscle was significantly elongated in the elderly after the intervention compared with before the intervention.

\section{cervical anterior inclination angle}

The increased anterior inclination of the cervical spine indicates the effectiveness of the verbal intervention in both standing and sitting positions. We compared our results with the reference value of cervical anteversion from other studies. The results showed that the cervical anterior inclination $90.67 \pm 9.67$ degree of the young people in our study was similar to that of Zhu et al. [21], while the cervical anterior inclination $91.34 \pm 9.92$ degree of the older people in our study was in contrast with that of Zhou [22]. In addition, the anterior inclination of the cervical spine of the sitting Young is the same as that of Zhou [22], which is different from what was reported by Hey [23]. This difference may be caused by differences in research methods and within-subjects, because there are obvious differences in the 
procedures and angle calculation methods of different photogrammetry methods [23], and even if the data collection methods in the study were similar, the calculation results could be very different [24].

Previous studies have shown that aging is associated with an increase in cervical anteversion relative to the horizontal direction [25]. Our study shows that the mean value of cervical anteversion is larger in the elderly than in the young, but there is no significant difference between the two groups. This may be caused by differences within the participants. However, Kado et al. found that, like ours, cervical kyphosis decreased significantly in both gender and age groups under 70 years old, and a significant decrease in cervical lordosis curvature was observed in women and young people [26]. In this study, due to the uneven gender distribution of the participants, the gender was not distinguished, but the correlation analysis showed that the cervical anteversion in standing position was significantly and negatively correlated with height, weight, and BMI, but the cervical anteversion in sitting position was not correlated to these anthropometric factors.

\section{Kyphosis angle of thoracic vertebrae}

The most obvious and recognizable postural change in the older adult group was an increase in thoracic kyphosis. Our results showed that the $25.42 \pm 5.80$ degree of thoracic kyphosis in the standing position in the older group was higher than that in the young group (23.28 \pm 4.11$)$, but there was no statistical significance. Hu's research results showed that the thoracic curvature of the elderly was $27.5 \pm 8.5$ degrees and the young group was $23.8 \pm 8.5$, which were similar to our reference value [27]. In addition, research by Kuo and colleagues also showed that there was no statistically significant difference between the age groups in standing positions [28]. The reason for this phenomenon may be that the older with a significant increase in thoracic kyphosis have other health conditions [13] and are excluded from the participants. In addition, studies have shown that there is a moderate positive correlation between age and thoracic kyphosis [29], which is consistent with the results of our correlation analysis.

Thoracic kyphosis is significantly reduced post the intervention in both young and older groups. Although the increase of thoracic kyphosis angle is related to aging, it can be changed through the adjustment of body posture. However, there was no statistical difference in thoracic kyphosis angle between sitting position and standing position. The result of the report in the literature is different from ours. Nishida found that the changes from standing to sitting posture flattened the kyphosis of the thoracic vertebrae [30], which may be caused by different postural standards or internal differences among the participants. In addition, interestingly, we found that the thoracic kyphosis angle in the sitting position was zero. This may be due to that, under the sitting position, the position of the pelvis on the supporting base was relatively fixed [31]. Without the cooperation of the anteversion of the pelvis, the thoracic vertebrae were straightened for compensation. Long-term fixed posture could lead to an increase in back muscle tension and a series of related disorders such as back pain [32].

\section{Lumbar lordosis angle}

A study has shown that aging is related to the decrease and loss of lumbar curvature [33]. The lumbar lordosis angle of the elderly is significantly smaller than that of the young, and the lumbar lordosis angle 
of the female is larger than that of the male [34]. The effect of BMI is not significant [35]. However, some researchers found that the reduction of these angles mainly occurred in the middle part of the kyphosis, less in the lumbosacral and thoracolumbar transition, and gender only affected the maximum range of upper body extension [36]. Therefore, the position of the marker point and the definition of the angle will also affect the result. When the same marker point is considered, the reference value of this study result can be used as a reference for body position evaluation in future studies [37].

Although our research also showed differences between the age groups ( $7.96 \pm 3.14$ degrees in the elderly, $9.85 \pm 5.73$ in the young), the results were not statistically significant. The results of Kuo were the same as ours. They found that the lumbar lordosis angle was 15. $2 \pm 9.3$ in the elderly and 16. $0 \pm 5.6$ in the young. There was no significant difference between the age groups [28]. In addition, our study was supported by Okpala et al., who found that the lumbar lordosis did not change significantly with age in the normal population [4]. Similarly, our correlation analysis did not show any significant correlation between age, $\mathrm{BMI}$, and lumbar lordosis angle.

In line with the purpose of our study, our results show that the lumbar lordosis angle was significantly increased Post-intervention. The change rate of lumbar flexure in the young and standing was $35.13 \%$, that in older and standing was $41.71 \%$, and that in young sitting was $40.28 \%$, which was higher than the intervention effect of other studies [38, 39]. However, an exception was from Berjano [40], that they reported the change rate of lumbar lordosis angle of $121.82 \%$.

The results of our study support the hypothesis that increasing the lumbar lordosis curvature by increasing the cervical anterior inclination angle. This practice could be used as an alternative to improving the lumbar curvature by using the pelvic forward inclination angle, especially under sitting conditions. Because the pelvis rotates backward when sitting, the psoas muscle tension decreases [41], and the lumbar lordosis decreases [42]. Schmidt and colleagues tried to improve lumbar curvature and relieve low back pain by correcting pelvic inclination, but the changes were not statistically significant [43]. In addition, whether in school or at home, school-age children spend more and more time in a sitting posture, and maintaining a sitting posture for a long time will lead to a decrease in lumbar lordosis and thoracic kyphosis [44]. Our study also supports this view, and the results show that the lumbar lordosis angle was significantly reduced in the sitting position compared with the standing position.

\section{Anteversion angle of the pelvis}

Our results showed that understanding posture, the pelvic forward inclination were significantly different between the two age groups, and the $22.97 \pm 5.01$ of young people was significantly higher than that of the older group $9.8 \pm 3.22$. In addition, the pelvic forward inclination angle was significantly and negatively correlated with age, and $\mathrm{BMI}$, and positively correlated with height. Hu et al. studied asymptomatic Chinese adults and found that the male pelvic inclination was greater than the female in all age groups, and the elder pelvic inclination was greater than the young (young 11.5 \pm 7.8 , older 14.5 \pm 9.5 )[45]. Another study reported that, in the standing position, the pelvic retroversion angle of the elderly volunteers was larger than that of the young people, and the pelvic retroversion occurred from standing to sitting position

Page $13 / 21$ 
[46]. These differences may be linked to age-related spinal degeneration because the thoracic kyphosis angle increases with age and is accompanied by a decrease in pelvic forward inclination to rebalance the spine.

There was a significant difference in pelvic forward inclination pre and post the intervention in standing posture. Some studies have shown that the increase of the anterior inclination of the pelvis is related to the decrease of the cervical angle [16], which is in line with our results, but because the location of the marker point of the cervical angle in this study is different from that study, when the cervical anterior inclination increases, the anteversion of the pelvis will also increase significantly. In addition, it is reported that the increase in pelvic forward inclination in the standing position may also be related to an increase in lumbar extension [37]. However, some researchers hold the opposite view as they found that the increase of pelvic forward inclination was related to the degree of lumbar lordosis when sitting, but had no relation with the degree of lumbar lordosis when standing [16]. Unfortunately, because of instrument limitations, in our study, we could not measure the pelvic inclination in the sitting position, which may be examined in future research.

\section{Limitations and future research direction}

Results of the current study can only apply to healthy people, should not be generalized to populations with back pain and other neuromuscular disorders. In addition, the sample size is relatively small and we could not examine the differences in gender.

Although several studies already explore the relationship between spine angles using external markers, there is still significant variation in the placement of the markers. To optimize the study of different spine angles and to facilitate comparison of the results between different studies, a more standardized method of spine angle analysis is needed.

Despite these limitations, as far as we know, this is still the first study aiming to improve the lumbar lordosis angle by adjusting the position of the head, which can provide a reference for the prevention and treatment of low back pain in the elderly and sedentary groups such as students.

\section{Conclusion}

The results of this study indicated that that increasing the cervical anterior inclination angle can indeed increase the lumbar lordosis angle, and reduce the thoracic kyphosis angle. Therefore, cervical anterior inclination can be used as an alternative to pelvic foreward inclination to improve the lumbar lordosis angle.

\section{Abbreviations}

LL: Lumbar lordosis; LBP: Low back pain; CAA: cervical anterior angle; LLA: Lumbar lordosis angle; TKA: thoracic kyphosis angle; PFIA: pelvic foreword inclination angle; ASIS: anterior superior iliac spine; PSIS: posterior superior iliac spine; 


\section{Declarations}

\section{Ethics approval and consent to participate}

Each author certifies that his or her institution has approved the protocol for any investigation involving humans or animals and that all experimentation was conducted in conformity with ethical and humane principles of research. Ethics Committee of Tianjin University of Sport (approval number TJUS2019032).

\section{Consent to publication}

All participants agreed to consent. Patients signed informed consent regarding publishing their data and photographs.

\section{Availability of data and materials}

The data in this study are available from the corresponding author upon request, if legally and ethically possible.

Each author warrants that his or her submission to the Work is original. Neither this Work nor a similar work has been published elsewhere in any language nor shall be submitted for publication elsewhere while under consideration by European Spine Journal.

\section{Competing interests}

The authors declare that they have no competing interests.

\section{Funding}

This research was financially supported by the National Natural Science Foundation of China, grant number 31271275.

\section{Authors' contributions}

$\mathrm{MZ}$ and $\mathrm{YH}$ : Design of the study, radiological assessments, data analysis and interpretation, and drafting of the manuscript. SZ: Conducted data collection, preliminary analyses and critical revision of the manuscript. JF and CP: Conducted data collection and preliminary analyses. LW: Contributed to analysis and interpretation of results, conclusions and dissemination. $\mathrm{MZ}$ and $\mathrm{YH}$ contributes equally to this work. All authors read and approved the final manuscript.

\section{Acknowledgements}

To the participants of this study who shared their time.

\section{References}


1. Wang XD, Ma L, Wang DH and Yan JT. Relationships among the lumbar lordosis index, sacral horizontal angle, and chronic low back pain in the elderly aged 60-69 years: A cross-sectional study. Journal of Back and Musculoskeletal Rehabilitation. 2019;33(2):1-5.

2. Kahere $M$ and Ginindza T. Mapping evidence on the prevalence, incidence, risk factors and cost associated with chronic low back pain among adults in Sub-Saharan Africa: a systematic scoping review protocol. Systematic Reviews. 2020;9(1):57.

3. Roussouly P and Pinheiro-Franco JL. Sagittal parameters of the spine: biomechanical approach. European Spine Journal 2011;20 Suppl 5:578-85.

4. Okpala FO. Measurement of lumbosacral angle in normal radiographs: A retrospective study in Southeast Nigeria. Annals of Medical and Health Sciences Research. 2014;4(5):757.

5. Pries $E$, Dreischarf $M$, Bashkuev M, Putzier $M$ and Schmidt $H$. The effects of age and gender on the lumbopelvic rhythm in the sagittal plane in 309 subjects. Journal of Biomechanics. 2015;48(12):3080-87.

6. De Carvalho D, Greene R, Swab M and Godwin M. Does objectively measured prolonged standing for desk work result in lower ratings of perceived low back pain than sitting? A systematic review and meta-analysis. [Journal Article; Meta-Analysis; Review; Systematic Review]. Work. 2020; 67(2):43140.

7. De Carvalho DE, Soave D, Ross K. and Callaghan J P. Lumbar Spine and Pelvic Posture Between Standing and Sitting: A Radiologic Investigation Including Reliability and Repeatability of the Lumbar Lordosis Measure. Journal of Manipulative and Physiological Therapeutics. 2010; 33(1):48-55.

8. Vujcic I, Stojilovic N and Dubljanin E, et al. Low Back Pain among Medical Students in Belgrade (Serbia):A Cross-Sectional Study. Pain Research and Management. 2018;https://doi.org/10.1155/2018/8317906

9. Merchant G, Buelna C and Castañeda SF, et al. Accelerometer-measured sedentary time among Hispanic adults: Results from the Hispanic Community Health Study/Study of Latinos (HCHS/SOL). Preventive Medicine Reports. 2015; 2:845-53.

10. Suzuki H, Endo K and Mizuochi J, et al. Sagittal lumbo-pelvic alignment in the sitting position of elderly persons. Journal of Orthopaedic Science. 2016;21(6):713-17.

11. Misir A, Kizkapan TB and Tas SK, et al. Lumbar spine posture and spinopelvic parameters change in various standing and sitting postures. European Spine Journal. 2019;28(5): 1072-81.

12. Claeys K., Brumagne S, Deklerck J, Vanderhaeghen J and Dankaerts W. Sagittal evaluation of usual standing and sitting spinal posture. Journal of bodywork and movement therapies. 2015;20(2): 32633.

13. Gogola A, Saulicz E, Kuszewski M, Matyja M and Myśliwiec A. Development of low postural tone compensatory patterns - predicted dysfunction patterns in upper part of the body. Developmental Period Medicine. 2014;18: 380-85.

14. Weisman MHS, Haddad M, Lavi N and Vulfsons S. Surface electromyographic recordings after passive and active motion along the posterior myofascial kinematic chain in healthy male subjects. 
Journal of Bodywork and Movement Therapies. 2014;18(3):452-61.

15. Ruivo RM, Pezarat-Correia P and Carita Al. Intrarater and interrater reliability of photographic measurement of upper-body standing posture of adolescents. Journal of Manipulative and Physiological Therapeutics. 2015;38(1):74-80.

16. Stolinski L, Kozinoga M and Czaprowski D, et al. Two-dimensional digital photography for child body posture evaluation: standardized technique, reliable parameters and normative data for age 7-10 years. Scoliosis and Spinal Disorders. 2017;12:38.

17. Candotti CT, Gelain GM and Antoniolli A, et al. Repeatability and Reproducibility of Postural Variables by Photogrammetry. Journal of Manipulative and Physiological Therapeutics. 2019;42(5):372-78.

18. Leroux MA, Zabjek $\mathrm{K}$ and Simard $\mathrm{G}$, et al. A noninvasive anthropometric technique for measuring kyphosis and lordosis: an application for idiopathic scoliosis. Spine. 2020;25(13): 1689-94.

19. Letafatkar A., Amirsasan R., Abdolvahabi Z and Hadadnezhad M. Reliability and validity of the AutoCAD software method in lumbar lordosis measurement. Journal of Chiropractic Medicine. 2011;10(4):240-47.

20. Finestone AS, Marcus G, Anekstein Y, Mirovsky Y and Agar G. Assessing kyphosis with SpineScan: another attempt to reduce our dependence on radiography. The spine journal: official journal of the North American Spine Society. 2013;13(8), 926-31.

21. Zhu Z, Xu L, F Zhu, et al. Sagittal Alignment of Spine and Pelvis in Asymptomatic Adults. Spine. 2014;39(1):E1-E6.

22. Zhou S, Li W, Wang W, et al. Sagittal Spinal and Pelvic Alignment in Middle-Aged and Older Men and Women in the Natural and Erect Sitting Positions: A Prospective Study in a Chinese Population. Medical Science Monitor. 2020;26:e919441.

23. Liu Ka-Po, Gabriel Teo, Alex \& Quok, et al. How the spine differs in standing and in sitting-important considerations for correction of spinal deformity. The spine journal : official journal of the North American Spine Society. 2017;17(6):799-806.

24. Porto AB and Okazaki V. Thoracic Kyphosis and Lumbar Lordosis Assessment by Radiography and Photogrammetry: A Review of Normative Values and Reliability. Journal of manipulative and physiological therapeutics. 2018;41(8):712-23.

25. Shin Y, Han K and Lee YH. Temporal Trends in Cervical Spine Curvature of South Korean Adults Assessed by Deep Learning System Segmentation, 2006-2018. JAMA network open. 2020;3(10):e2020961.

26. Kado DM. Hyperkyphosis Predicts Mortality Independent of Vertebral Osteoporosis in Older Women. Annals of Internal Medicine. 2009;150(10):681.

27. Hu L, Lv Y and Lin Y. Correlations and Age-Related Changes of Cervical Sagittal Parameters in Adults Without Symptoms of Cervical Spinal Disease. Spine. 2020;45(23):E1542-E1548.

28. Kuo Y, Tully EA and Galea MP. Video Analysis of Sagittal Spinal Posture in Healthy Young and Older Adults. Journal of Manipulative and Physiological Therapeutics. 2009;32(3):210-15. 
29. Iyer S, Lenke LG, Nemani VM, et al. Variations in Sagittal Alignment Parameters Based on Age. Spine. 2016; 41(23):1826-36.

30. Nishida N, Izumiyama T, Asahi R, et al. Changes in the global spine alignment in the sitting position in an automobile. The spine journal : official journal of the North American Spine Society. 2020;20(4):614-20.

31. Fortin C, Ehrmann Feldman D, Cheriet F, et al. Differences in Standing and Sitting Postures of Youth with Idiopathic Scoliosis from Quantitative Analysis of Digital Photographs. Physical \& Occupational Therapy In Pediatrics. 2013;33(3):313-26.

32. Maigne JY, Cornelis $P$ and Chatellier G. Lower back pain and neck pain: is it possible to identify the painful side by palpation only? Annals of physical and rehabilitation medicine. 2012; 55(2):103-11.

33. Amonoo-Kuofi US. Changes in the Lumbosacral Angle, Sacral Inclination and the Curvature of the Lumbar Spine during Aging. Cells Tissues Organs. 1992;145(4):373-77.

34. Coskun $\mathrm{BI}$ and Basaran $\mathrm{S}$. Comparative study of lumbosacral alignment in elderly versus young adults: data on patients with low back pain. Aging Clinical and Experimental Research. 2015;27(3): 297-02.

35. Youdas JW, Hollman JH and Krause DA. The effects of gender, age, and body mass index on standing lumbar curvature in persons without current low back pain. Physiotherapy theory and practice. 2006;22(5):229-37.

36. Marcel D, Laia A, Antonius R, et al. Age-Related Loss of Lumbar Spinal Lordosis and Mobility - A Study of 323 Asymptomatic Volunteers. PLoS ONE. 2014;9(12): e116186.

37. Krawczky B, Pacheco AG and Mainenti MR. A systematic review of the angular values obtained by computerized photogrammetry in sagittal plane: a proposal for reference values. Journal of manipulative and physiological therapeutics. 2014;37(4): 269-75.

38. Lee JH, Lee DO, Lee JH, et al. Effects of Lordotic Angle of a Cage on Sagittal Alignment and Clinical Outcome in One Level Posterior Lumbar Interbody Fusion with Pedicle Screw Fixation. BioMed Research International. 2015;https://doi.org/10.1155/2015/523728

39. Berglund, Lars, Aasa, et al. Sagittal lumbopelvic alignment in patients with low back pain and the effects of a high-load lifting exercise and individualized low-load motor control exercises-a randomized controlled trial. The Spine Journal. 2018;18(3): 399-06.

40. Berjano P, Pejrona M, Damilano M, et al. Corner osteotomy: a modified pedicle subtraction osteotomy for increased sagittal correction in the lumbar spine. European Spine Journal 2015;24 Suppl 1:58-65.

41. Been E and Kalichman L. Lumbar Lordosis. Spine. 2014;14(1):87-97.

42. Kusakabe T, Endo $K$, Aihara T, et al. Differences in cervical sagittal alignment between the standing and sitting positions. Journal of Orthopaedic Science. 2019;24(6):1005-09.

43. Schmidt S, Wlfle N, Schultz C, et al. Assessment of a taping method combined with manual therapy as a treatment of non-specific chronic low back pain-a randomized controlled trial. BMC Musculoskeletal Disorders. 2021;22(1):410. 
44. Drza-Grabiec J, Truszczyńska A, Ma GF, et al. Changes of the body posture parameters in the standing versus relaxed sitting and corrected sitting position. Journal of Back and Musculoskeletal Rehabilitation. 2016; 29(2):211-17.

45. Hu Zongshan,Man Gene Chi Wai,Yeung Kwong Hang et al. 2020 Young Investigator Award Winner: Age- and Sex-related Normative Value of Whole-body Sagittal Alignment Based on 584 Asymptomatic Chinese Adult Population From Age 20 to 89 .Spine (Phila Pa 1976). 2020;45: 79-87.

46. Zhou S, Sun Z, Li W, et al. The standing and sitting sagittal spinopelvic alignment of Chinese young and elderly population: does age influence the differences between the two positions? European Spine Journal. 2020;29 Suppl 6:405-12.

\section{Figures}

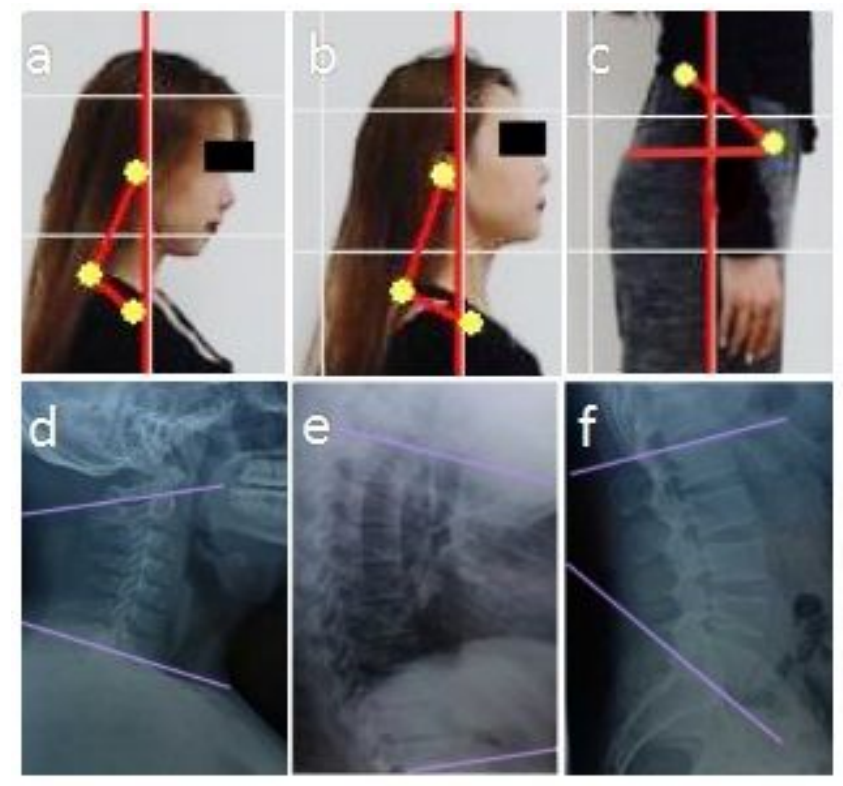

\section{Figure 1}

a Standing CAA. The definition of CAA is the angle between two lines with Tragus of Ear and Spinous process of $\mathrm{C} 7$ and Spinous process of $\mathrm{C} 7$ and greater tuberosity of humerus. $\mathbf{b}$ Raising CAA. The definition of raising CAA is the same as the standing CAA. c PFIA. Definition of PFIA is the ASIS-PSIShorizontal plane. $\mathbf{d}$ Sitting CAA. Definition of Sitting CAA is the angle between lower endplates of $\mathrm{C} 1$ and that of C7. e TKA. Definition of TKA is the angle between T4 superior endplates and T12 lower endplates. $\mathbf{f}$ LLA. Definition of LLA is the angle between superior endplates of L1 and that of S1. 


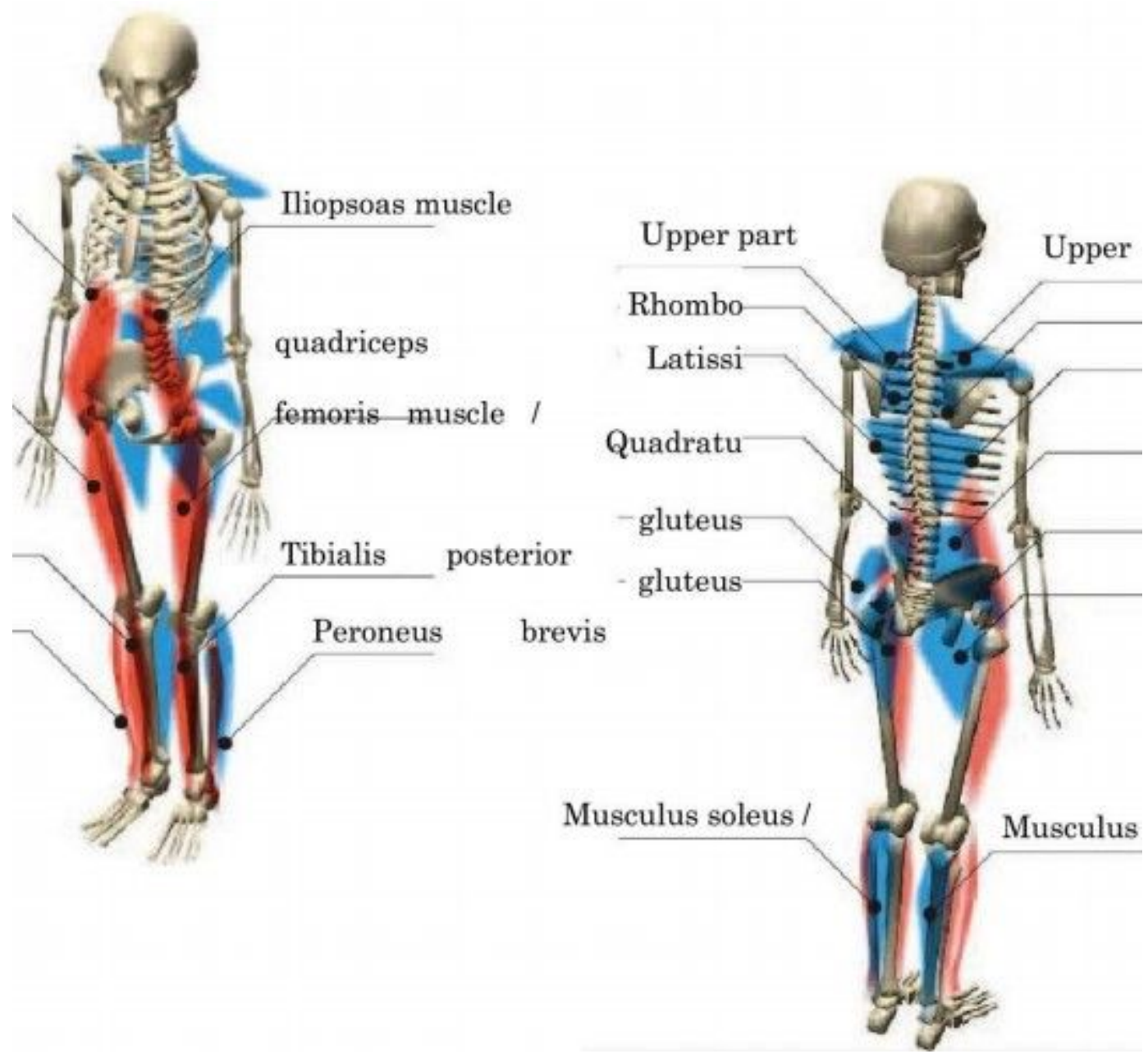

Figure 2

Muscle tension simulation image (generated by PA200) 

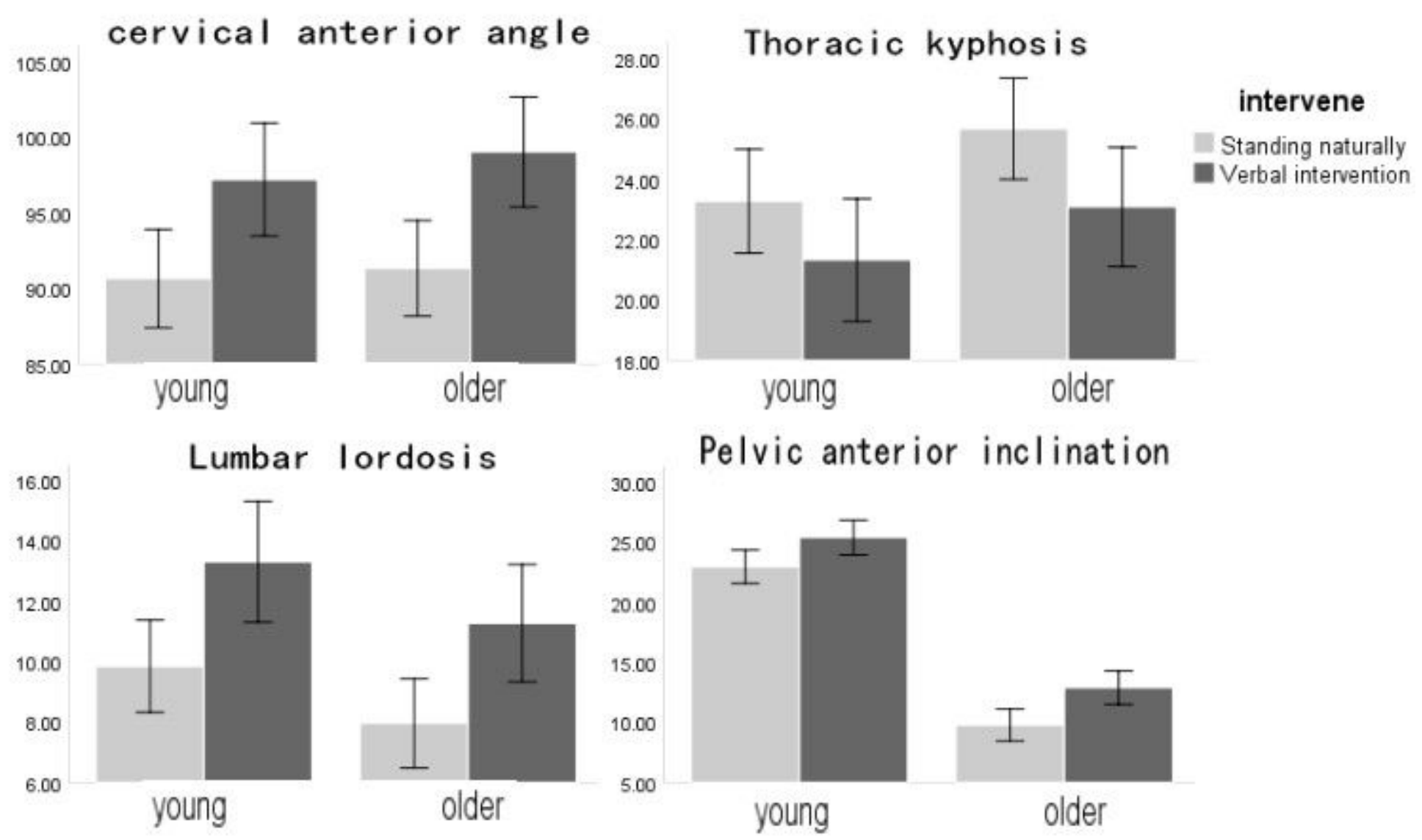

Figure 3

Comparison of the spinal angles between the young and older adults in a standing position (Unit: degree) (Gray represents pre the postural cueing and black represents post the postural cueing)
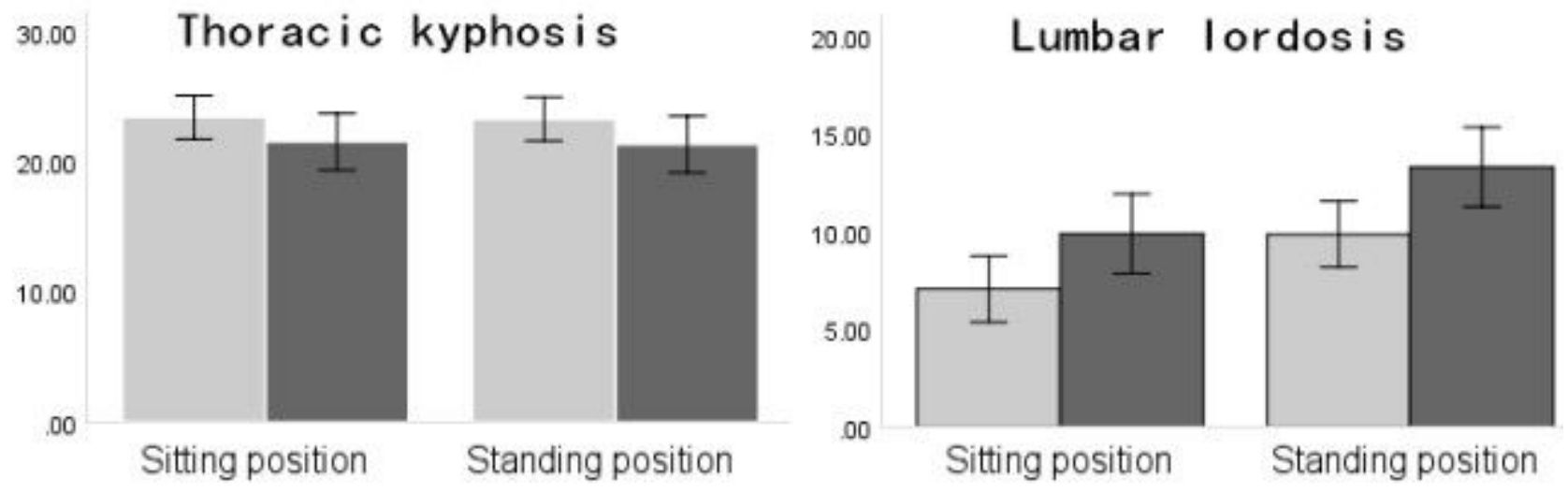

\section{Figure 4}

Comparison of sitting and standing position, TKA and LLA in young adults (Unit: degree) (Gray represents pre the postural cueing and black represents post the postural cueing) 